\title{
Absolute Orientation of Molecules at Interfaces
}

\author{
Yi Rao, Matthew Comstock, and Kenneth B. Eisenthal* \\ Department of Chemistry, 3000 Broadway, Columbia University, New York, New York 10025
}

Received: September 20, 2005; In Final Form: November 7, 2005

A method to determine the absolute orientation of molecules at liquid interfaces by sum frequency generation (SFG) is reported. It is based on measurements of the orientations of two nonparallel vibrationally active chromophores in the molecule of interest combined with a rotation matrix formulation to obtain the absolute molecular orientation. We chose $m$-tolunitrile, a planar molecule adsorbed to the air/water interface, as a proof-of-method experiment. Quantitative analysis of different polarization sum frequency intensities facilitate unique peak assignments of the methyl and nitrile groups of $m$-tolunitrile. The SFG analysis of the measurement yields a nitrile group tilting at $53^{\circ}$ to the surface normal, and the $C_{3}$ axis of the methyl group is almost upright at $23^{\circ}$ with respect to the surface normal. Using a rotation matrix formulation, we found that the angle between the surface plane and the $m$-tolunitrile molecular plane is $70^{\circ}$.

\section{Introduction}

Knowledge of molecular orientation at surfaces and interfaces is of widespread interest to scientists because of its relevance to chemical reactions, long- and short-range molecular interactions, phase transitions, and the electronic and vibrational states of interfacial molecules. ${ }^{1-3}$ Because the molecular interface is only a few molecules thick, interfacial populations are very small. As a consequence, a useful optical technique must be interface specific and sensitive to composition, orientation, and structure at the interface. Second harmonic generation (SHG) and sum frequency generation (SFG), as second-order interactions, can be employed to specifically investigate interfaces that are accessible to light, including buried interfaces., ${ }^{2,3}$ SHG depends primarily on the electronic properties of the interfacial molecules, whereas SFG is sensitive to molecular vibrations when one of the incident beams is in the infrared. Traditionally, SFG has been performed by scanning the incident IR light point by point and mixing it with visible light of picosecond or nanosecond duration. A new approach to the measurement of vibrational spectra using SFG (broadband SFG) has been developed and has proven to be successful. ${ }^{4}$ It entails combining a spectrally broad IR pulse, which is characteristic of femtosecond IR pulses, with a spectrally narrow visible pulse. ${ }^{4}$ In the frequency-resolved SFG experiment, the bandwidth of the incident IR pulse is much larger than that of the vibrational resonance. The frequency-resolved sum frequency signals cover several hundred wavenumbers, which makes it possible to more rapidly obtain vibrational spectra of interest. The spectral resolution results from the spectrally narrow $\left(10 \mathrm{~cm}^{-1}\right)$ picosecond visible laser pulse.

With the recent developments in laser technology, SFG is widely used not only in fundamental studies of interfaces, but also in technological studies of catalysis, chemical vapor deposition, electrochemistry, polymers, and environmental interfaces..$^{2,3,5-8}$ Orientation measurements of chemical groups at interfaces and surfaces were realized soon after the SFG technique was applied to the study of interfaces and surfaces. ${ }^{9}$ Recently, the orientational angle with respect to the surface

* To whom correspondence should be addressed. E-mail: eisenth@ chem.columbia.ed. normal and the twist angle relative to the plane comprising the surface normal and the symmetry axis of the isopropyl headgroup of a leucine molecule at the air/water interface was measured. ${ }^{10}$ However, a complete determination of the absolute orientation of this entire molecule or other molecules has not been reported.

The basic idea of the method reported here is to measure the orientations of two SFG active chromophores with respect to the surface normal to obtain the absolute orientation defined by both symmetry axes and the molecular plane with respect to the surface normal. Combining these measured values with the known orientations of the chromophores with respect to each other in the molecule together with a rotation matrix operation formulation, we obtain the absolute orientation of the interfacial molecule. Generally, the alignment of the molecule at the interface, that is, which part of the molecule projects toward which bulk phase, is known. If not, phase measurements will provide the up versus down alignment. We chose $m$-tolunitrile, which is a planar molecule having two distinct vibrational chromophores, as a proof-of-method example to demonstrate how to obtain the absolute orientation of the molecule at the interface.

\section{Theoretical Considerations}

Here we shall briefly outline the fundamental theory of SFG as it applies to surfaces and interfaces to facilitate the discussion that follows. We consider the case of two incident lasers, one infrared and one visible, neither of which is one- or two-photon resonant with excited electronic states.

The sum frequency intensity is proportional to the square of the sum over all macroscopic sum frequency susceptibility terms separated into a nonresonant part, $\chi^{(2), \mathrm{NR}}$, and a resonant part, $\chi^{(2), \mathrm{R}}(\omega)$.

$$
\begin{gathered}
I^{\mathrm{SF}}(\omega) \propto\left|\chi^{(2), \mathrm{NR}}+\sum_{q}^{n} \chi^{(2), \mathrm{R}}(\omega, q)\right|^{2} \\
\chi^{(2), \mathrm{R}}(\omega, q) \propto \frac{A_{q}}{\omega_{\mathrm{ir}}-\omega_{q}+i \Gamma_{q}}
\end{gathered}
$$


where $A_{q}$ contains the product of the Raman and IR matrix elements of the $q$ th normal mode, $\omega_{q}$ denotes the resonant frequency of the $q$ th vibrational normal mode, $\omega_{\text {ir }}$ is the frequency of the IR light, and $\Gamma_{q}$ is the spectral width of the vibrational state. In the case of dielectric interfaces, the contribution from the nonresonant parts is small compared to the resonant terms of interest.

Here we define the $X Y$ plane in the laboratory coordinate system $(X, Y, Z)$ as the plane of the interface, the $Z$ axis as the surface normal, and $Y Z$ as the plane of incidence. Among the total 27 macroscopic susceptibility tensors $\chi_{I J K}^{(2)}(I, J$, and $K$ being the three laboratory coordinates), there are 7 nonzero terms for a rotationally isotropic interface ( $C_{\infty v}$ symmetry), namely, $\chi_{X X Z}^{(2)}=\chi_{Y Y Z}^{(2)} ; \chi_{X Z X}^{(2)}=\chi_{Y Z Y}^{(2)} ; \chi_{Z X X}^{(2)}=\chi_{Z Y Y}^{(2)} ;$ and $\chi_{Z Z Z}^{(2)}$. In our experiments the $Y Z$ plane was the plane of incidence for both the visible and IR beams. The two polarization combinations measured in each experiment were $S S P$ and $P P P$, where the first letter denotes the polarization of the sum frequency, the second one denotes the polarization of the visible light, and the last one denotes the polarization of the IR light. These polarizations are connected with the laboratory coordinates by nonlinear Fresnel coefficients and appropriate projections. ${ }^{11-13}$ The seven nonzero macroscopic susceptibilities in the laboratory coordinates are obtained from the four polarization combinations.

We approximate the macroscopic sum frequency susceptibility tensors $\chi_{I J K}^{(2), R}(\omega)$ in the laboratory coordinates $(X, Y, Z)(I, J$, and $K$ being the three coordinates) with the microscopic hyperpolarizability tensor elements $\beta_{i j k}^{(2)}$ in the molecular coordinates $(x, y, z)(i, j$, and $k$ being the three coordinates $)$ yielding

$$
\chi_{I J K}^{(2)}=N_{\mathrm{s}}\langle(\hat{I} \cdot \hat{\imath})(\hat{J} \cdot \hat{j})(\hat{K} \cdot \hat{k})\rangle \beta_{i j k}^{(2)}=N_{\mathrm{s}}\left\langle\hat{R}_{I \cdot i} \hat{R}_{J \cdot j} \hat{R}_{K \cdot k}\right\rangle \beta_{i j k}^{(2)}
$$

where $N_{\mathrm{s}}$ is the number density of the interface moiety under investigation, $\hat{R}$ is the element of the rotation transformation matrix from the molecular frame to the laboratory frame, and \langle\rangle denotes the ensemble average over the orientation of the interfacial molecules of interest.

In the theory of vibrational SFG with a single resonance at the IR frequency, the tensor elements of the hyperpolarizability are related to the IR and Raman properties of the vibrational mode, and are given by the partial derivative of the Raman polarizability tensor and the IR transition dipole moment of the $q$ th mode with respect to the normal coordinate of the same mode. ${ }^{14}$ It is given by the following equation:

$$
\beta_{i j k, q}^{(2)}=\frac{1}{2 \epsilon_{0} \omega_{q}} \frac{\frac{\partial \alpha_{i j}}{\partial Q_{q}} \frac{\partial \mu_{k}}{\partial Q_{q}}}{\omega_{\mathrm{ir}}-\omega_{q}+i \Gamma_{q}}
$$

where $\partial \alpha_{i j} / \partial Q_{q}$ is the derivative of the Raman polarizability element, and $\partial \mu_{k} / \partial Q_{q}$ is the derivative of the transition dipole moment for the $q$ th normal mode.

To determine orientational structure, we chose to use the null angle method in our data analysis rather than the intensity ratio method. A comparison of the two methods applied to both SHG and SFG orientational analysis was carried out. ${ }^{12,15}$ It was found that the primary disadvantage for the intensity ratio method is that PPP or SSP SFG intensity is often small, which, as a consequence, makes it difficult to obtain an accurate intensity ratio from direct SFG intensity measurements. Nonetheless, it is to be noted that there are vibrations for which the intensity ratio method is satisfactory. In the experiments reported here, the null angle method is used to obtain five nonvanishing

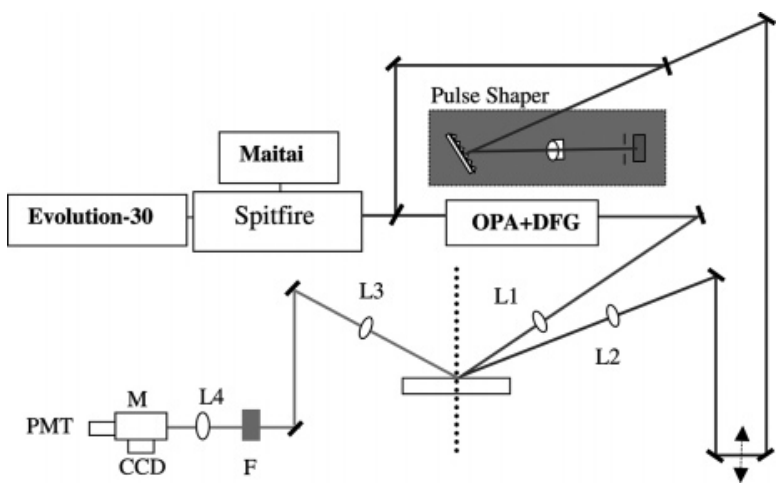

Figure 1. The layout of the broadband SFG system. L1: $10 \mathrm{~cm} \mathrm{FL}$ CaF2 lens; L2: $20 \mathrm{~cm}$ FL silica lens; L3: $20 \mathrm{~cm}$ FL BK7 lens; L4: $15 \mathrm{~cm}$ FL BK7 lens. The pulse shaper consists of grating, a $15 \mathrm{~cm}$ FL cylindrical lens, and a high-reflectivity dielectric mirror. F: dichroic mirrors; M: monochromator; PMT: photomultiplier tube; CCD: charge-coupling device.

elements by analyzing the SFG intensities at different detection polarization angles.

\section{Experimental Section}

3.1 Laser. An $800 \mathrm{~nm}$ regeneratively amplified Ti:sapphire system (Spitfire, Spectra Physics) seeded with a MaiTai 80 $\mathrm{MHz}, 80 \mathrm{fs}$ oscillator, at a $1 \mathrm{kHz}$ repetition frequency was employed in the present experiments. A reflected $20 \%$ output from the regenerative amplifier was used to produce a picosecond $800 \mathrm{~nm}$ pulse. A signal generated by the input of the $800 \mathrm{~nm}$ femtosecond pulse into an optical parametric amplification (OPA) was in the range of $1.1-1.6 \mu \mathrm{m}$, and the idler was in the range of 1.6-2.8 $\mu \mathrm{m}$. By difference frequency generation in a $1 \mathrm{~mm} \mathrm{AgGaS2}$ crystal, tunable IR light between $3 \mu \mathrm{m}$ and $8.5 \mu \mathrm{m}$ with a bandwidth of about $150 \mathrm{~cm}^{-1}$ (fwhm) was generated.

Figure 1 is a schematic experimental setup of the system. The reflected femtosecond beam from the regenerative amplifier was spectrally narrowed using a home-built pulse shaper to provide an $800 \mathrm{~nm}$ pump pulse with a bandwidth of $10 \mathrm{~cm}^{-1}$ and a time duration of $2.5 \mathrm{ps}$, which was determined from temporal cross-correlation measurements with the femtosecond $800 \mathrm{~nm}$ laser. The output power after pulse shaping was typically $17 \mu \mathrm{J}$ per pulse. The IR beam is focused onto the sample by a $\mathrm{BaF} 2$ lens with a $100 \mathrm{~mm}$ focal length (FL) at an angle of $67^{\circ}$ relative to the surface normal and a spot size of around 120 $\mu \mathrm{m}$. The visible light field is focused to $210 \mu \mathrm{m}$ spot size by a BK glass lens with a $250 \mathrm{~mm}$ FL at an angle of $76^{\circ}$ from the surface normal.

A $0.5 \mathrm{~m}$ spectrograph with one entry and two exits (Acton Research, three gratings, including 1200 grooves/mm with 450 $\mathrm{nm}$ blazed, 1200 grooves $/ \mathrm{mm}$ with $500 \mathrm{~nm}$ blazed, and 600 grooves $/ \mathrm{mm}$ with $4 \mu \mathrm{m}$ blazed) was used. The $500 \mathrm{~nm}$ blazed grating was chosen to measure sum frequency signals from the methyl and the nitrile groups, and the $4 \mu \mathrm{m}$ blazed grating was used for an IR intensity measurement. One exit was configured for detecting the dispersed signal with a liquid-nitrogen-cooled, back-thinned charge-coupled device (CCD) camera (Roper Scientific, $1340 \times 400$ pixels) operating at $-120{ }^{\circ} \mathrm{C}$.

3.2 Sample Preparation. The purity of $m$-tolunitrile was checked by gas chromography and NMR and used without further purification. Its chemical structure is shown in the inset of Figure 2. The samples used in this work were at $6 \mathrm{mM}$. From the surface tension measurements in Figure 2, the surface area occupied per molecule of $m$-tolunitrile at $6 \mathrm{mM}$ was found to 


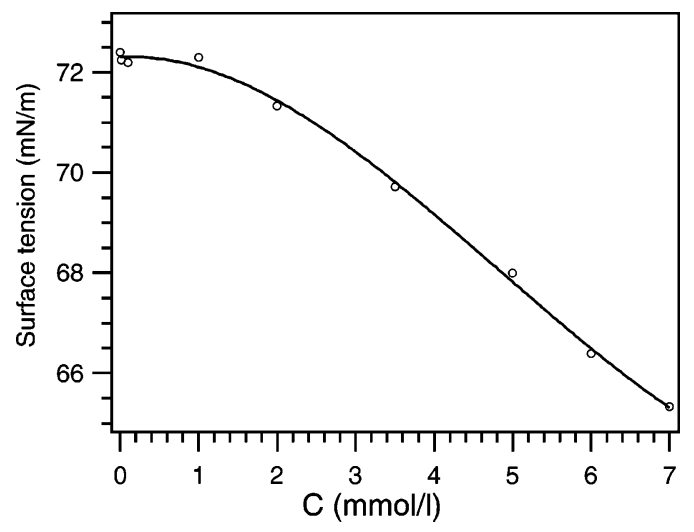

Figure 2. The surface tension of $m$-tolunitrile aqueous solutions at different $m$-tolunitrile concentrations.

be $56 \AA^{2}$. To avoid thermal effects, the sample holder was placed on a rotating stage at $4 \mathrm{rpm}$ in all experiments. The experiments were performed at $21^{\circ} \mathrm{C}$.

\section{Results and Discussion}

When thinking about the orientation of $m$-tolunitrile at the air/aqueous solution, it is reasonable to assume that the hydrophilic nitrile group preferentially projects into the bulk water, as was found in SFG studies of various hydrocarbon nitriles at the air/aqueous nitrile solution, whereas the hydrophobic methyl group is out of the water. In this discussion, we have defined the so-called "left-handed screw" to define the Euler transformation between the laboratory coordinates $(X, Y$, $Z)$ and the molecular coordinates $(x, y, z) .{ }^{16} \mathrm{We}$ adopted the center of the ring as the origin $(O)$ of all coordinates. The direction from the nitrogen toward the carbon is designated as positive $z$, the $x$ axis is perpendicular to the molecular plane, and the $y$ axis is in the molecular plane. We define the direction of the unit vector from the origin $O$ toward the carbon of the $\mathrm{C}-\mathrm{CH}_{3}$ to be along the $\mathrm{Oz}^{\prime}$ axis. The orientational angle of the nitrile group and the methyl group with respect to the surface normal are designated as $\theta_{1}$ and $\theta_{2}$, respectively. We shall assume $\delta$-function distributions for orientational angles. From the orientational angles of the two groups with respect to the surface normal, together with a rotation matrix formalism to obtain the angle between the normal to the phenyl plane and the normal to the interface, we obtained the absolute orientation of $m$-tolunitrile.

4.1 Nitrile Group of $m$-Tolunitrile at the Air/Aqueous Interface. Figure 3 shows the SFG spectra of a $6 \mathrm{mM}$ $m$-tolunitrile aqueous solution taken at two different polarization combinations, SSP and PPP, with the visible beam at $800 \mathrm{~nm}$ and the IR centered at $4.7 \mu \mathrm{m}$ with a bandwidth of $140 \mathrm{~cm}^{-1}$. From fits of the SFG spectrum using two Lorentzian functions, the peak at $2234 \mathrm{~cm}^{-1}$ in the $S S P$ polarization spectrum was assigned to the symmetric stretching mode of $-\mathrm{CN}$, with a shoulder at $2270 \mathrm{~cm}^{-1}$, which becomes more prominent in the $P P P$ spectrum. We considered three possibilities in the assignment of the shoulder at $2270 \mathrm{~cm}^{-1}$. One possibility is that the molecules form aggregates at the interface. To check this, we diluted the $m$-tolunitrile bulk concentration by a factor of 2 and found no change in the spectrum. The SFG intensity decreased by a factor of 4 , which is expected for bulk concentrations that are in the linear part of the $m$-tolunitrile adsorption isotherm. We conclude that aggregation is not responsible for the 2270 $\mathrm{cm}^{-1}$ shoulder. Another possibility is that the nitrile group of $m$-tolunitrile is hydrogen bonded with water. ${ }^{17,18}$ We performed

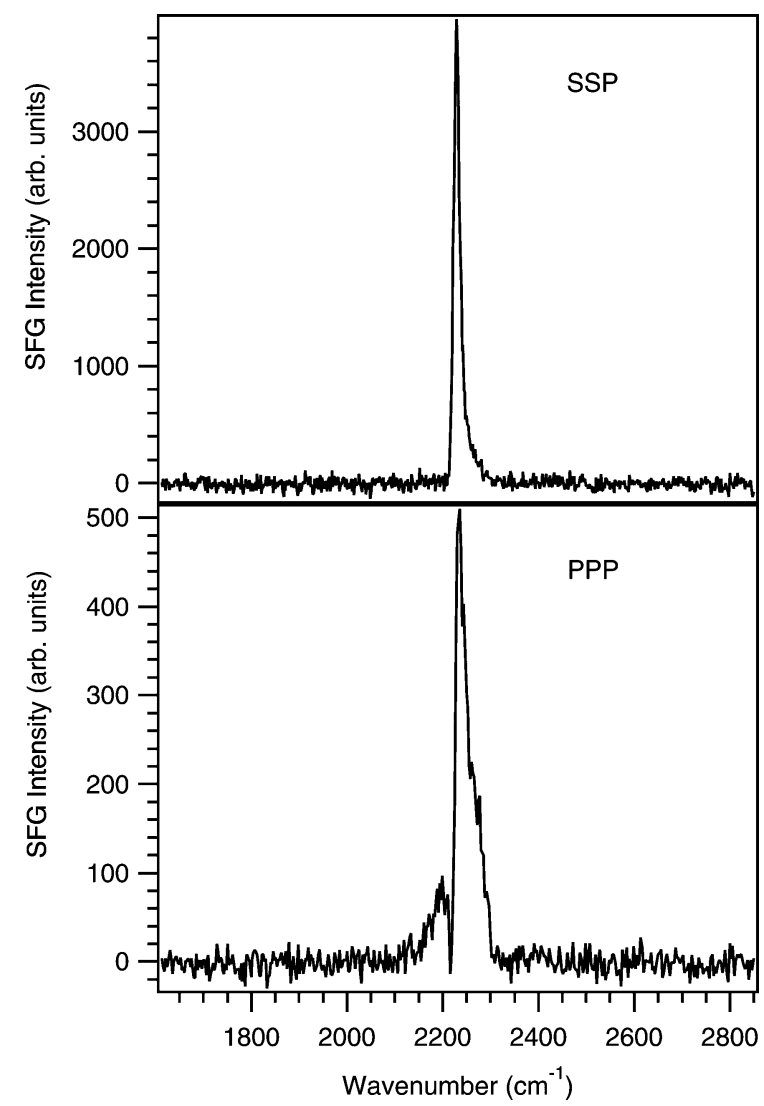

Figure 3. The nitrile $(-\mathrm{CN}) S S P$ and $P P P$ sum frequency spectra of $6 \mathrm{mM} m$-tolunitrile with visible light at $800 \mathrm{~nm}$ and the IR centered at $4.7 \mu \mathrm{m}$.

Raman experiments with $6 \mathrm{mM} m$-tolunitrile aqueous solution and neat $m$-tolunitrile and found that the two Raman spectra are the same with only one peak at $2224 \mathrm{~cm}^{-1}$ (the data are not shown here); noting, however, that hydrogen bonding of the $-\mathrm{CN}$ with water can differ at the interface compared with that in the bulk aqueous nitrile solution. The third possibility is that the $2270 \mathrm{~cm}^{-1}$ shoulder is a combination band of some modes in the $1000-1200 \mathrm{~cm}^{-1}$ energy range. ${ }^{18,19}$ In the $P P P$ spectrum, we observed the peak at $2234 \mathrm{~cm}^{-1}$, the shoulder at $2270 \mathrm{~cm}^{-1}$, and a weak peak at $2205 \mathrm{~cm}^{-1}$. The weak peak at $2205 \mathrm{~cm}^{-1}$ is attributed to the overtone of the $\mathrm{C}-\mathrm{H}$ in-plane bending mode of the phenyl ring of $m$-tolunitrile at $1100 \mathrm{~cm}^{-1} .{ }^{20} \mathrm{We}$ have found that there is a strong Raman peak at $1100 \mathrm{~cm}^{-1}$ in both neat $m$-tolunitrile and $6 \mathrm{mM} m$-tolunitrile aqueous solution. The SPS and PSS spectra for the $-\mathrm{CN}$ stretching mode were not detected under our experimental setup, which is consistent with our simulated results described later.

There are three nonvanishing elements for the $C_{\infty v}$ symmetry of $-\mathrm{CN}$, namely, $\beta_{x x z}=\beta_{y y z}=r \beta_{z z z}$, where $r$ is $\beta_{x x z} / \beta_{z z z}$. From eq 3 we see that $r=\alpha_{x x}^{\text {Raman }} / \alpha_{z z}^{\text {Raman }}$, that is, the ratio of the Raman perpendicular polarizability element to the Raman parallel polarizability element of the $-\mathrm{CN}$ bond. From Raman polarization measurements, two possible ratios are obtained; which one is correct cannot be determined from the Raman measurements alone. However, as has been shown in previous work, a determination of the relative sign of $\chi_{x x z}^{(2)}$ and $\chi_{x z x}^{(2)}$ from SFG measurements can be used to identify the correct value of the polarizability ratio $r$, which is $0.26 .1^{17,21}$ To obtain the orientation of the $-\mathrm{CN}$ chromophore, null angle measurements of the nitrile stretch mode were performed. The measured intensity plotted against the polarization angle of the analyzer is shown in Figure 4. The null angle $\Omega_{\mathrm{CN}}$ can be fitted from 


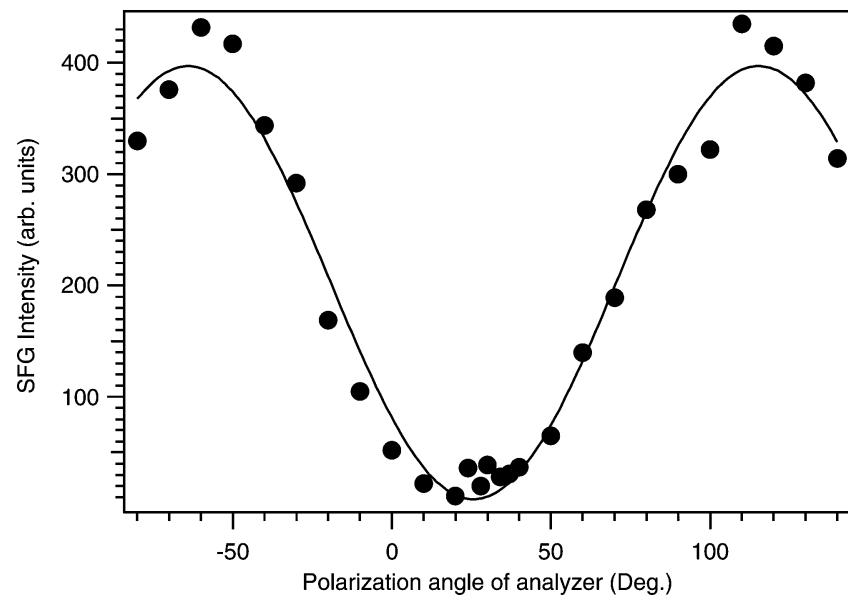

Figure 4. SFG intensity of the nitrile $(-\mathrm{CN}) 2234 \mathrm{~cm}^{-1}$ peak plotted against the polarization angle of the analyzer. The solid line is the calculated fit.

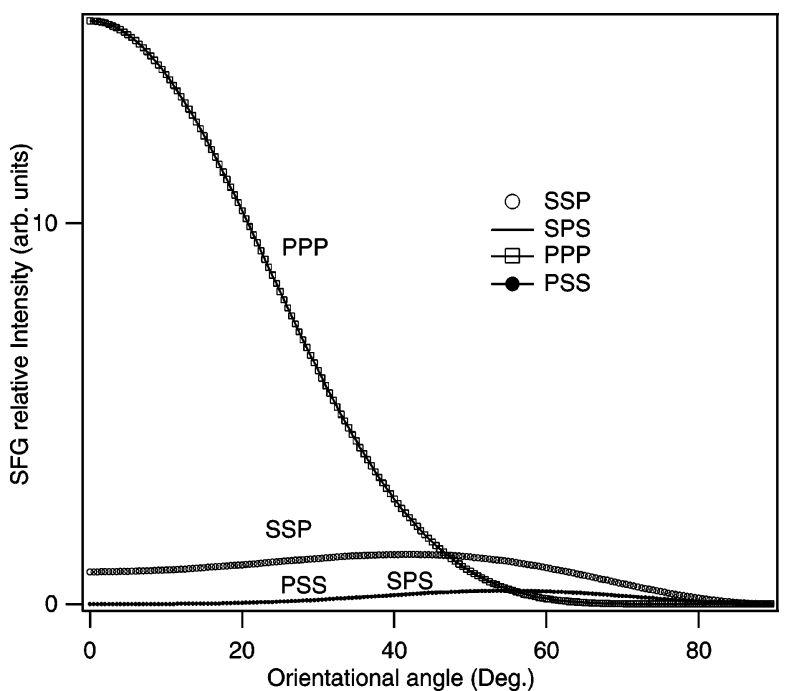

Figure 5. The numerically calculated SFG relative intensities of the nitrile group under four different polarization combinations ( $S S P, P P P$, $S P S$, and $P S S)$ vs the orientation of the nitrile group $(-\mathrm{CN})$ with respect to the interface normal. The refractive index of the $m$-tolunitrile aqueous solution and monolayer are set to be 1.40 and 1.20 , respectively, at all wavelengths.

Figure $4,{ }^{15,22,23}$ from which we found that the orientational angle $\theta_{1}$ of the nitrile group is $53.0 \pm 1.3^{\circ}$ with respect to the surface normal.

We also calculated the orientational angle using the intensity ratio method with the intensity of $P P P$ and SSP polarization. The orientational angle was found to be $51.0 \pm 2.1^{\circ}$, which is in good agreement with the orientational angle obtained with the null angle method. To assist us in the interpretation of our results, we performed numerical calculations to obtain the relative intensities of the four polarization combinations using a specific calculation procedure ${ }^{24}$ Briefly, we considered the macroscopic susceptibilities and their connections with the microscopic hyperpolarizabilities under different polarization combinations. Calculations of the relative intensities of the generally used polarization combinations SSP, PPP, SPS, and $P S S$ show the relative intensities as a function of the orientational angle in Figure 5. For the SPS and PSS polarizations, the relative intensities are less than that in the case of $P P P$ polarization, which brings them below our detection limit. This is consistent with our experimental findings that we did not observe SFG signals for the SPS and PSS polarization combinations.

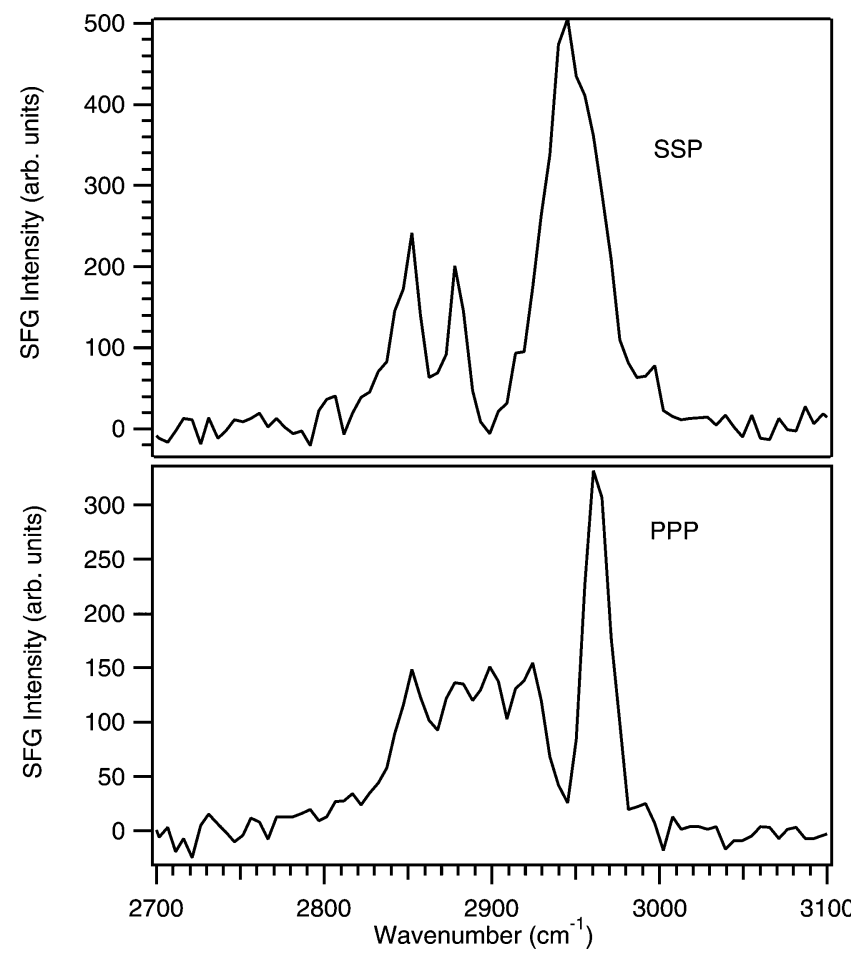

Figure 6. The methyl group $\left(-\mathrm{CH}_{3}\right) S S P$ and $P P P$ sum frequency spectra of a $6 \mathrm{mM} m$-tolunitrile aqueous solution with the visible light at $800 \mathrm{~nm}$ and the IR centered at $3.4 \mu \mathrm{m}$.

4.2 Methyl Group Vibration of $\boldsymbol{m}$-Tolunitrile at the Air/ Aqueous Interface. Figure 6 shows the SFG spectra of $m$-tolunitrile at the air/aqueous interface taken at two different polarization combinations, SSP and $P P P$, with the visible wavelength at $800 \mathrm{~nm}$ and the IR wavelength centered at 3.4 $\mu \mathrm{m}$ with a bandwidth of $150 \mathrm{~cm}^{-1}$. The $P P P$ polarization combination spectrum shows a strong peak centered at 2961 $\mathrm{cm}^{-1}$. On the red side of the peak, there are three weak peaks at 2853,2878 , and $2898 \mathrm{~cm}^{-1}$. In the case of the $S S P$ polarization spectrum, there is a strong peak centered at $2945 \mathrm{~cm}^{-1}$ and two weaker features at 2853 and $2878 \mathrm{~cm}^{-1}$. The strong peak at $2945 \mathrm{~cm}^{-1}$ in the SSP SFG spectrum is assigned to the symmetric stretch of the methyl group,,$^{25,26}$ and the $2961 \mathrm{~cm}^{-1}$ peak of the PPP spectrum was assigned to the antisymmetric stretch mode. ${ }^{25,26}$ For the aromatic methyl group of a disubstituted benzene, the two peaks at 2853 and $2878 \mathrm{~cm}^{-1}$ are assigned to overtones of $\mathrm{CH}_{3}$ antisymmetric bending vibrations or to the mixing of $\mathrm{C}-\mathrm{C}$ stretching and $\mathrm{C}-\mathrm{H}$ bending modes of the phenyl ring. ${ }^{20,27}$ The small feature at $2898 \mathrm{~cm}^{-1}$ can result from the Fermi resonance of the methyl symmetric stretch mode with the overtone of the $\mathrm{CH}_{3}$ antisymmetric bending mode.

A plot of the sum frequency intensity versus the polarization angle of the analyzer at $2945 \mathrm{~cm}^{-1}$ is shown in Figure 7. For the symmetric stretch mode, two nonvanishing elements of the hyperpolarizabilities exist, namely, $\beta_{x x z}=\beta_{y y z}=r \beta_{z z z}$, where the ratio $r$ value of 2.3 was used by combining the Raman depolarization of the methyl group in acetonitrile with the SFG measurements of the relative sign of the $\chi_{X X Z}^{(2)}$ and $\chi_{X Z X}^{(2)}$ susceptibility elements. ${ }^{17}$ Using the null angle method, the calculated orientational angle of the $C_{3}$ axis of the methyl group is $23.0 \pm 1.6^{\circ}$. The refractive index of the $m$-tolunitrile aqueous solution and monolayer are set to be 1.40 and 1.20 , respectively, at all wavelengths. We also employed the intensity ratio of the $S S P$ and PPP spectrum at the $2945 \mathrm{~cm}^{-1}$ peak to calculate the orientational angle of the methyl group and found the value to 


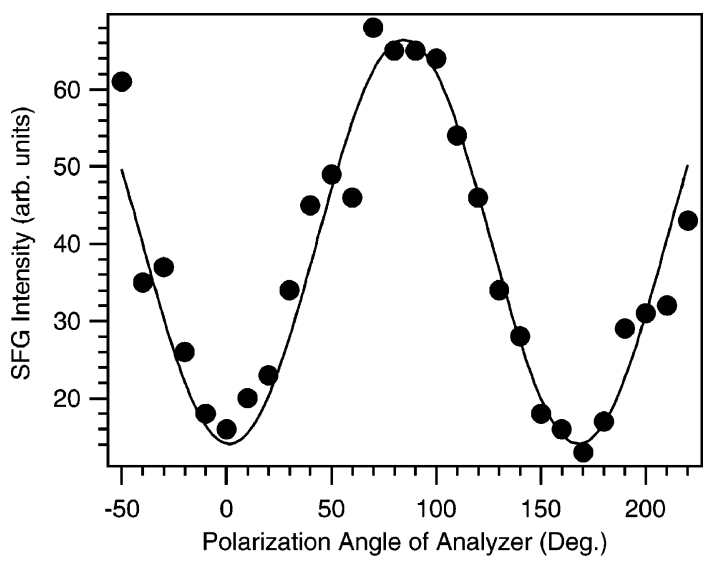

Figure 7. SFG intensity of the methyl group $\left(-\mathrm{CH}_{3}\right) 2945 \mathrm{~cm}^{-1}$ peak plotted against the polarization angle of the analyzer. The solid line is the calculated fit.

be $28^{\circ} \pm 1.2^{\circ}$, which is close to the results from the null angle measurements.

4.3 The Absolute Orientation of $m$-Tolunitrile at the Air/ Aqueous Solution. Now that we have obtained the orientational angle $\theta_{1}$ of the nitrile group and the orientational angle $\theta_{2}$ of the methyl group, we can determine the absolute orientation of the $m$-tolunitrile molecule at the air/aqueous interface. ${ }^{16}$ To extract the remaining angle needed to determine the absolute orientation of $m$-tolunitrile, we performed two rotation operations. The remaining angle to be determined involves the orientation of the phenyl group, which we define as the angle between the normal to the phenyl plane and the interface normal. The two rotation operations relate the laboratory axes $(X, Y, Z)$ and the molecular axes $(x, y, z)$. Initially, we set the molecular plane in the $Z Y$ plane of the laboratory coordinates, the symmetry axis of the $-\mathrm{CN}$ is along both the $z$ molecular axis and the $Z$ laboratory axis (negative), and, finally, the $X$ and $x$ axes are perpendicular to the molecular plane, as shown in Figure 8A. Thus, in the laboratory coordinates, the initial coordinates of the methyl group $C_{3}$ axis expressed in terms of the direction cosines with the laboratory axes $\left(l_{\mathrm{CH}_{3}, X}^{0}, l_{\mathrm{CH}_{3}, Y}^{0}\right.$, $\left.l_{\mathrm{CH}_{3}, \mathrm{Z}}^{0}\right)$ is $\left(0, \cos 30^{\circ}, \cos 60^{\circ}\right)$.

As the first step, the molecular coordinates are rotated by an angle of $\theta_{1}=53^{\circ}$ around the $X$ axis such that the orientational angle of the nitrile group with respect to the surface normal is $53^{\circ}$ as obtained from null angle measurements (Figure $8 \mathrm{~B}$ ). This rotation operation $R_{X}\left(\theta_{1}\right)$ changes the spatial coordinates of the methyl group $C_{3}$ axis from its initial orientation $\left(0, \cos 30^{\circ}\right.$, $\left.\cos 60^{\circ}\right)$ to the orientation $\left(l_{\mathrm{CH}_{3}, X}^{1}, l_{\mathrm{CH}_{3}, Y}^{1}, l_{\mathrm{CH}_{3}, \mathrm{Z}}^{1}\right)$ of the methyl group after the first rotation operation $R_{X}\left(\theta_{1}\right)$, where $\theta_{1}=53^{\circ}$.

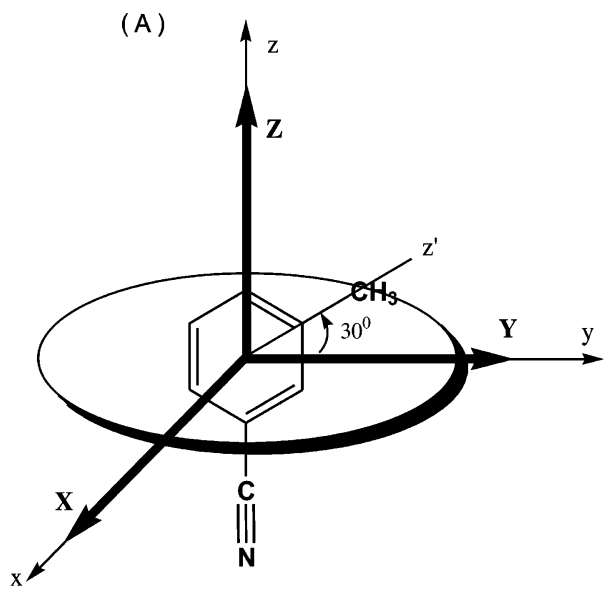

(B)
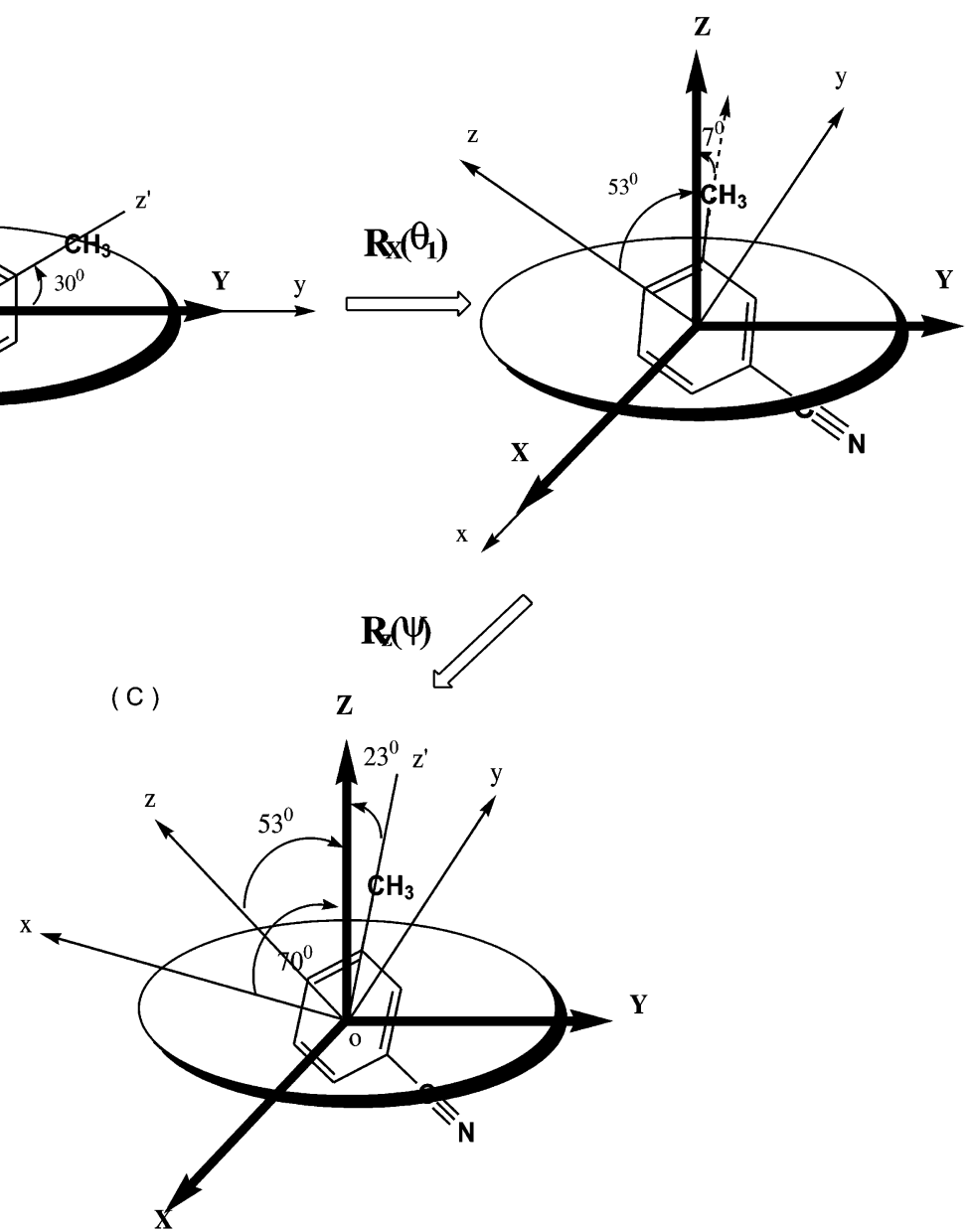

Figure 8. The rotation operations used to obtain the orientation of the molecular plane from the measured orientations of the $-\mathrm{CN}$ and $-\mathrm{CH}_{3}$ groups. (A) The initial configuration of the $m$-tolunitrile in the laboratory coordinates with the $Z$ axis along the $-\mathrm{CN}$ symmetry axis and the $X$ axis perpendicular to the molecular plane. (B) The molecular configuration after the first rotation operation of $53^{\circ}$ (measured orientation of $-\mathrm{CN}$ with respect to the interface normal $Z$ ) around the laboratory $X$ axis. (C) The final molecular configuration after the second rotation around the molecular $z$ axis, bringing the $C_{3}$ axis of the $\mathrm{CH}_{3}$ group to its measured orientation of $23^{\circ}$ with respect to the interface normal $Z$. The normal to the molecular plane, given by the molecular coordinate $x$, is found to be $70^{\circ}$ with respect to the surface normal. 
This rotation operation can be written as

$$
\begin{aligned}
\left(\begin{array}{l}
l_{\mathrm{CH}_{3}, X}^{1} \\
l_{\mathrm{CH}_{3}, Y}^{1} \\
l_{\mathrm{CH}_{3}, \mathrm{Z}}^{1}
\end{array}\right) & =R_{X}\left(\theta_{1}\right) \cdot\left(\begin{array}{l}
l_{\mathrm{CH}_{3}, X}^{0} \\
l_{\mathrm{CH}_{3}, Y}^{0} \\
l_{\mathrm{CH}_{3}, \mathrm{Z}}^{0}
\end{array}\right)= \\
& \left(\begin{array}{lll}
1 & 0 & 0 \\
0 & \cos \theta_{1} & -\sin \theta_{1} \\
0 & \sin \theta_{1} & \cos \theta_{1}
\end{array}\right) \cdot\left(\begin{array}{l}
0 \\
\cos 30^{\circ} \\
\cos 60^{\circ}
\end{array}\right)=\left(\begin{array}{l}
0 \\
\cos 83^{\circ} \\
\cos 7^{\circ}
\end{array}\right)
\end{aligned}
$$

The methyl group $C_{3}$ axis initially at $30^{\circ}$ with respect to the $Y$ axis is now at an angle of $83^{\circ}$ with respect to the $Y$ axis and $7^{\circ}$ with respect to the $Z$ axis after a rotation of $53^{\circ}$ about the $X$ axis. For the nitrile group, the rotation is simple and will be shown later.

To bring the angle of the methyl group $C_{3}$ axis with respect to the surface normal $Z$ to its measured value of $23^{\circ}$, a rotation of $\psi$ degrees about the molecular $z$ axis $(-\mathrm{CN})$ is performed (Figure $8 \mathrm{C}$ ). The mathematical representation of the rotation of the methyl about the molecular $z$ axis can be expressed as a product of rotations about the laboratory $X$ and $Z$ axes. ${ }^{16}$ This leads to a final expression

$$
R_{z}(\psi) \cdot R_{X}\left(\theta_{1}\right) \cdot\left(\begin{array}{l}
0 \\
\cos 30^{\circ} \\
\cos 60^{\circ}
\end{array}\right)=\left(\begin{array}{l}
l_{\mathrm{CH}_{3}, X}^{\mathrm{final}} \\
l_{\mathrm{CH}_{3}, Y}^{\mathrm{final}} \\
l_{\mathrm{CH}_{3}, \mathrm{Z}}^{\text {fina }}
\end{array}\right)=\left(\begin{array}{l}
\cos 112^{\circ} \\
\cos 86^{\circ} \\
\cos 23^{\circ}
\end{array}\right)
$$

Since the $-\mathrm{CN}$ bond remains in the $Y Z$ plane in the laboratory coordinates under this rotation, the orientation of the $-\mathrm{CN}$ axis in the laboratory coordinates $\hat{e}_{\mathrm{CN}}$ is $\left(0, \cos 37^{\circ},-\cos 53^{\circ}\right)$. Note that the carbon is closer to the origin than the nitrogen, and the $-\mathrm{CN}$ bond is located at a negative $z$ coordinate.

By taking the cross product of the unit vector $\hat{e}_{\mathrm{CH}_{3}}$ corresponding to the methyl orientation $\left(l_{\mathrm{CH}_{3}, X}^{\mathrm{final}}, l_{\mathrm{CH}_{3}, Y}^{\mathrm{final}}, l_{\mathrm{CH}_{3}, Z}^{\mathrm{final}}\right)$, with the unit vector $\hat{e}_{\mathrm{CN}}$ corresponding to the $-\mathrm{CN}$ orientation $\left(l_{\mathrm{CN}, X}^{\mathrm{final}}, l_{\mathrm{CN}, Y}^{\mathrm{final}}, l_{\mathrm{CN}, Z}^{\mathrm{fina}}\right)$, we obtain a vector $\hat{e}_{x}$, which is normal to the molecular plane, and $\hat{e}_{x}=\hat{e}_{\mathrm{CH}_{3}} \times \hat{e}_{\mathrm{CN}}$ is expressed as direction cosines $\left(\cos 39^{\circ}, \cos 77^{\circ}, \cos 73^{\circ}\right)$. We can now calculate the molecular orientation, assuming that the molecules are isotropically distributed in the surface $X Y$ plane. By projecting the unit vector $\hat{e}_{x}$ onto the laboratory $Z$ axis, we can calculate the angle $\varphi$ of the normal to the molecular plane $\hat{e}_{x}$ and the surface normal $\hat{Z}$, which is also the angle between the molecular plane and the surface plane.

$$
\varphi=\cos ^{-1}\left(\hat{e}_{x} \cdot \hat{Z}\right)=70^{\circ}
$$

Combining the orientation of the $-\mathrm{CN}$, the methyl $C_{3}$ axis and the molecular plane, we can depict the $m$-tolunitrile at the air/aqueous interface, as shown in Figure 8C.

\section{Conclusions}

In this paper, we have performed a proof-of-concept experiment demonstrating that measurements of the orientation of two nonparallel chromophores in the molecule of interest when combined with a rotation matrix formulation yield the absolute molecular orientation at the interface. The orientations of the nitrile group $(-\mathrm{CN})$ and the methyl $\left(-\mathrm{CH}_{3}\right) C_{3}$ axis of $m$-tolunitrile at the air/water interface were obtained with SFG measurements of these IR and Raman active vibrational chromophores. The SFG measurements showed that, with respect to the surface normal, the nitrile group tilts at $53^{\circ}$, the $C_{3}$ axis of the methyl group tilts at $23^{\circ}$, and, combining these results with a two-rotation operation procedure, we find that the angle between the molecular and surface planes is $70^{\circ}$.

Acknowledgment. This work was supported by the National Science Foundation and the Chemical Sciences, Geosciences and Biosciences Division, Office of Basic Energy Sciences, Office of Science, U.S. Department of Energy. Y.R. thanks Dr. Feng Wang, Dr. Na Ji, and Daohua Song for beneficial discussions. The authors also thank Professor Hong-fei Wang for his valuable discussions.

\section{References and Notes}

(1) Adamson, A. Physical Chemistry of Surfaces, 5th ed.; John Wiley and Sons: New York, 1990.

(2) Shen, Y. R. Annu. Rev. Phys. Chem. 1989, 40, 327-350.

(3) Eisenthal, K. B. Chem. Rev. 1996, 96, 1343-1360.

(4) Richter, L. T.; Petralli-Mallow, T. P.; Stephenson, J. C. Opt. Lett 1998, 23, 1594-1596.

(5) Bain, C. D. J. Chem. Soc., Faraday Trans. 1995, 91, 1281-1296.

(6) Richmond, G. L. Chem. Rev. 2002, 102, 2693-2724.

(7) Chen, Z.; Shen, Y. R.; Somorjai, G. A. Annu. Rev. Phys. Chem. 2002, 53, 437-465.

(8) Shultz, M. J.; Schnitzer, C.; Simonelli, D.; Baldelli, S. Int. Rev. Phys. Chem. 2000, 19, 123-153.

(9) Zhu, X. D.; Suhr, H. J.; Shen, Y. R. J. Opt. Soc. Am. B 1986, 3, $\mathrm{P} 252$

(10) Ji, N.; Shen, Y. R. J. Chem. Phys. 2004, 120, 7107-7112.

(11) Zhuang, X.; Miranda, P. B.; Kim, D.; Shen, Y. R. Phys. Rev. B 1999, 59, 12632-12640.

(12) Rao, Y.; Tao, Y. S.; Wang, H. F. J. Chem. Phys. 2003, 119, 52265236.

(13) Wei, X.; Hong, S. C.; Zhuang, X. W.; Goto, T.; Shen, Y. R. Phys. Rev. E 2000, 62, 5160-5172.

(14) Superfine, R.; Huang, J. Y.; Shen, Y. R. Chem. Phys. Lett. 1990 172, 303-306.

(15) Lu, R.; Gan, W.; Wang, H. F. Chin. Sci. Bull. 2003, 48, 21832187

(16) Goldstein, H. Classical Mechanics, 2nd ed.; Addison-Wesley: Reading, MA, 1980

(17) Zhang, D.; Gutow, J.; Eisenthal, K. B. J. Phys. Chem. 1994, 98 , 13729-13734.

(18) Kryachko, E. S.; Nguyen, M. T. J. Chem. Phys. 2001, 115, 833-

(19) Seiichi Ishikawa, T. E.; Mikami, N. J. Chem. Phys. 1999, 110, 9504-9515.

(20) Varsanyi, G.: Szoke, S. Vibrational Spectra of Benzene Derivatives; Academic Press: New York and London, 1969.

(21) Yakovenko, S. Y.; Maiwald, M.; Wurflinger, A.; Pelzl, J. Liq. Cryst. $1999,26,23-30$.

(22) Hicks, J. M.; Kemnitz, K.; Eisenthal, K. B. J. Phys. Chem. 1986, $90,560-562$.

(23) Lu, R.; Gan, W.; Wu, B. H.; Chen, H.; Wang, H. F. J. Phys. Chem. B 2004, 108, 7297-7306

(24) Wang, H. F.; Gan, W.; Lu, R.; Rao, Y.; Wu, B. H. Int. Rev. Phys Chem. 2005, 24, 191-256.

(25) Bell, G. R.; Li, Z. X.; Bain, C. D.; Fischer, P.; Duffy, D. C. J. Phys. Chem. B 1998, 102, 9461-9472.

(26) Hommel, E. L.; Allen, H. C. Analyst 2003, 128, 750-755.

(27) Green, J.; Harrison, D. Spectrochim. Acta 1976, 32A, 1279- 